methods used in determining optical wave-lengths seemed almost hopeless. It was not until 1912, when Laue suggested that the regular spacings of the atoms in a crystal might serve as a naturally occurring diffraction grating for X-rays, that real progress was made. The suggestion was immediately verified by Friedrich and Knipping, and in the following year W. L. Bragg, by a most ingenious combination of crystallography and optical theory, succe日ded in determining simultaneously the grating constant of a rock-salt crystal, and the wave-length of the X-radiation employed.

This achievement has a double significance. If the spacing of the atoms in a crystal is known, we can, from diffraction measurements, determine the wavelength of the radiation impinging upon it, and thus obtain an X-ray spectrum. Conversely, if the wavelength of the radiation is known, we can deduce the spacings or lattice constants of the crystal. Thus, in the X-ray spectrometer, crystallography has found a most powerful research tool which has given quite a new impetus to this rather old-fashioned science. The practical importance of X-ray erystallography in metallurgy can scarcely be over-estimated. Since each chemical compound has its own unique set of lattice constants, it is possible to identify, not merely the elements present in a given substance (a task for which ordinary spectroscopy or chemical analysis will suffice), but also the actual compounds which these elements form with each other, and the temperatures at which these compounds form, or at which they disappear. When the field has been fully surveyed, and the $\mathrm{X}$-ray crystallographic index which is "already in active preparation is completed, it will be possible to identify any crystalline compound in an alloy or mixture by X-ray analysis. Nor is the field limited to true crystals. Many long-chain organic substances are sufficiently regular in their make-up to act as X-ray gratings, and much information, valuable both on the theoretical and the industrial side, has already been obtained (to quote only one example) on the structure of the fibres used in the weaving of textiles. To understand why substances behave as they do, in terms of the position of the atoms within them, is the first step in a scientific search for new and better materials; and X-ray crystallography seems destined to play a part in industry even more important than in radiography.

To attempt to survey the part played by X-rays in atomic research would involve the recital of the greater part of the history of that part of the subject dealing with the structure of the electron atmosphere which surrounds the central nucleus and on which most of the chemical and physical properties of the atom depend. Most of our exact knowledge-and it is very exact-of the arrangement, or to be more precise, the energy-levels, of the electrons in the atom, is derived from $\mathrm{X}$-ray data. It may be recalled that Moseley's early survey of the characteristic X-ray spectra given out by the elements provided the first clear demonstration of the fundamental importance of the idea of atomic number; that is to say, the charge upon the atomic nucleus as opposed to the atomic weight, which was shown to be of only secondary importance. It is interesting to notice that Moseley's results showed that four elements still remained to be discovered, and that two of these have since been identified by their X-ray spectra. It may also be mentioned that the most accurate determinations of the two fundamental physical constants, the charge on an electron and Planck's constant, are, in all probability, those derived from $\mathrm{X}$-ray measurements.

The temptation to moralize on the history of the discovery of $\mathbf{X}$-radiation, and its consequences, is strong; but the task may be left to the reader. Nature is full of surprises, and it is not always the most obviously desirable researches which yield the richest harvest. The lone experimenter who is prepared to follow his own inspiration, even if it seems to lead him away from the main current of research, has often played an important part in the progress of science; and pure science in particular, and society in general, owe much to the inspired curiosity of Wilhelm Konrad Röntgen.

\section{DIFFRACTION METHODS IN MODERN STRUCTURAL CHEMISTRY}

$\mathrm{N}$ the Tilden Lecture delivered before the Chemical I Society on January 18, Prof. J. M. Robertson outlined first the scope, limitations and possible future developments of the X-ray and electron diffraction methods, and then went on to consider the nature and lengths of bonds with particular reference to the results of recent diffraction studies. The emphasis throughout was on organic structures, as would be expected from one who has contributed more than any other single worker to our knowledge of the precise crystal structures of organic compounds.

In comparing the electron and X-ray diffraction methods, a number of points were brought out. The great value of the former is that it is applicable to gases and the vapours of easily volatile substances, which cannot conveniently be studied by the X-ray method. In this sense the two methods are complementary. We might add that, as regards inorganic compounds, they are complementary in another sense. In general, the same finite molecules exist in the vapour as in the crystals of an organic compound, but this is not usually so in inorganic chemistry. Electron diffraction studies of the vapours of many metallic salts and of some of the compounds of nonmetals give information about the structures of molecules which do not exist in the crystalline material.

A comparison was made of the nature of the experimental data obtainable by the two methods and of the ways in which they are interpreted. To the eye the electron diffraction photograph shows merely a number of rings on a background of decreasing intensity, and their positions and intensities are estimated visually. The X-ray photograph from a single crystal, on the other hand, shows a large number of discrete spots, and the positions and intensities of these can be determined with consider. able accuracy. This apparent advantage of the X-ray method is, however, offset by two complications. First, the molecules in the gas scatter independently of one another and they are oriented in all possible ways. This makes it possible to calculate the diffraction effects to be expected for any given molecular model and to compare them with those observed, the parameters in the model being varied until agreement is obtained. In the case of diffraction by a crystal, this cannot be done. In the crystal the molecules are definitely oriented with respect to one another, so that not only has the molecular model to be varied 
but also the mutual orientations, and hence the intermolecular distances. Secondly, it is usually impossible to utilize more than a small fraction of the X-ray data on the photographs. Apart from purely technical difficulties, which can eventually be overcome, there is a complication inherent in the $\mathrm{X}$-ray method, namely, that the structure amplitude $F_{h k l}$ corresponding to a particular reflexion is a complex quantity with an amplitude and a phase constant. From the observed intensity we can in general determine only the amplitude. Except in certain cases, therefore, it is necessary to work by a trial and error method based on a probable molecular model which is progressively refined as the structure-determination proceeds. The difficulty of determining phase constants may sometimes be overcome by making comparisons of data from isomorphous compounds or by studying crystals containing heavy atoms. If we assume complete ignorance of phase constants, then all the X-ray data are summarized on a Patterson vector diagram which is directly derived from the observed intensities. An analogy was drawn by Prof. Robertson between the Patterson method for X-ray diffraction and the radial distribution method for electron difiraction. Apart from the difficulty of interpreting Patterson diagrams, owing to the overlapping of peaks, there is the more fundamental difficulty that the same vector diagram (and hence the same X-ray diffraction pattern) may arise from different arrangements of atoms. The discussion of the uniqueness of the solutions of diffraction problems may appear to some chemists rather academic, especially as Prof. Robertson emphasized that a structure must always be consistent with all the other available physical and chemical evidence. However, while it is true that in the case of simple structures there is seldom any doubt as to the correct interpretation of the data, it is important, as the compounds studied become more and more complex, to re-examine the fundamental theory of all methods of interpreting diffraction data.

When discussing possible future developments, Prof. Robertson emphasized the need both for making fuller use of the experimental data and for improving its range and quality. $\mathrm{He}$ pointed out that many of the difficulties now encountered in making precise analyses are not fundamental, "and there is no doubt that they can be overcome, largely by the proper planning and organisation of the research, which will have to be on a fairly large scale". Although hopeful that diffraction methods will eventually give information about the positions of hydrogen atoms and about electron densities in bonds, he was careful to indicate some of the difficulties of interpreting the finer details on electron-density maps. On the whole, it would seem advisable to defer drawing conclusions from two-dimensional electron-density projections, and possibly to make detailed studies of the effect of temperature on electron-density distributions in selected crystals using sections through threedimensional summations, in order to discover the effect of the thermal movements of the molecule as a whole and of different parts of the molecule relative to one another. More emphasis might have been placed on an essential difference between electron and $\mathrm{X}$-ray diffraction, namely, that the nuclei are more effective than the orbital electrons in scattering high-speed electrons, whereas the scattering of X-rays is due to the orbital electrons alone. It is difficult to see how the electron diffraction method in its present form can provide information comparable with the electron-density maps derived from X-ray studies of crystals. Also, just as the thermal vibrations in solids result in the blurring of these maps, so the greater flexibility of some molecules in the vapour state makes it difficult, or even impossible, to obtain a complete picture of the molecule by the electron diffraction method. For example, the Sn-I distance in $\mathrm{SnI}_{2}$ can be determined, but not the I-Sn-I bond angle. Provided the molecule possesses sufficient rigidity, however, quite complex structures may now be studied by the electron diffraction method, as shown by the recent work on pirylene, diphenylene and tri-(phosphonitrile chloride).

Diffraction methods not only provide information about the general configurations of molecules but also they lead to accurate determinations of interatomic distances and inter-bond angles. The X-ray method; of course, gives data on intermolecular as well as intramolecular bonds, and although the weak van der Waals bonds are of comparatively little interest to the chemist, much valuable information about hydrogen bonds has been obtained from studies of crystals. A feature of modern structural chemistry is the detailed analysis of data on bond-lengths with the object of discovering more about the nature of the bonds. At one time 'chemical' bonds were regarded as either ionic or covalent, and in the latter case as single, double or triple bonds. The present view is that all bonds, other than those between atoms of the same element, have some ionic character and also that bonds intermediate between single and multiple bonds exist in many molecules. Bonds of these intermediate types are described in terms of resonance between structures with different arrangements of bonding electrons.

Prof. Robertson discussed the 'order' of carboncarbon bonds. The C-C distances for pure single-, double- and triple-bonds are well established; 1.54 A. in diamond, $1 \cdot 34 \mathrm{~A}$. in ethylene and $1.20 \mathrm{~A}$. in acetylene. Assuming 50 per cent double-bond character in benzene $(\mathrm{C}-\mathrm{C}=1.39 \mathrm{~A}$.) and 33.3 per cent in graphite (C-C = 1.42 A.), Pauling, Brockway and Beach constructed a curve relating percentage double-bond character to bond-length, from which can be estimated the amounts of double-bond character of other bonds with lengths between 1.54 and $1 \cdot 34 \mathrm{~A}$. The more detailed calculations of 'bond order' which predict minor variations in length among the bonds in molecules such as naphthalene and coronene cannot yet be checked as the differences are of the same order of magnitude as (or less than) the present experimental errors. This illustrates the need for more precise determinations of interatomic distances. On the other hand, there appear to be some abnormal bond-lengths which cannot at present be accounted for theoretically.

Dealing with bonds between unlike atoms, Prof. Robertson mentioned the recent revisions of certain of the Pauling-Huggins radii, so long adopted as the basis for discussions of interatomic distances. Observed distances which were less than the sums of these radii were considered exceptional, and elaborate explanations in terms of partial ionic character and partial double-bond character have been put forward. With the new (higher) values for the covalent radii of nitrogen, oxygen and fluorine, many bond-lengths are now seen to be appreciably less than the sums of the appropriate covalent radii, even where there is no likelihood of partial double-bond character. The empirical relationship of Schomaker and Stevenson attributes these differences to the ionic character 
of the bond, and uses the electronegativity coefficients of Pauling to correct the sums of the Pauling-Huggins radii. In other words, the fact that bonds are shorter than the sums of the true covalent radii is on this later view assumed to be due in many cases to the partial ionic rather than the partial double-bond character of the bonds. In view of the importance now to be attached to Pauling's electronegativity coefficients in discussions of bond type, it is well to remember that our difficulties in this field are an indication that we have not so far solved one of the most complex and fundamental problems in chemistry.

The early electronic theory of valency assigned electronic formulæ to many molecules, but offered no explanation either of the observed inter-bond angles or of the differences in properties between atoms with the same number and arrangement of valency electrons. As regards bond angles, it was necessary to find a way of investigating the 'group properties' of a number of valency electrons, and this problem has been solved to some extent by the methods of wavemechanics. We are still, however, far from being able to account for the chemical properties of an atom. Although elements of a group such as the halogens, all with similar sets of outermost electrons, have certain characteristics in common, nevertheless each halogen has a distinct 'individuality' and the changes in properties do not all run parallel with the increase in size (or atomic number). Thus although the properties of the atoms are to some extent determined by their outer electronic structures, the finer differences between atoms which are similar in this respect will not be explained until we are able to take into account the effect of the nuclear charge and the remaining shells of electrons.

Until we find some theoretical way of specifying these distinctive chemical properties in terms of the structure of the isolated atom, they can only be estimated from the way in which the atom interacts with other atoms. The use of the electronegativity concept represents an attempt to deal with the problem in this way. The electronegativity coefficients are derived from experimental observations on the interactions between atoms, namely, from bond energies. The new empirical relation connecting actual bond lengths with true covalent radii and electronegativity coefficients simply expresses the fact that the difference between the length of a bond $A-B$ and the arithmetic mean of $A-A$ and $B-B$ is related to the difference between the energy of the bond $A-B$ and the arithmetic mean of the energies of the bonds $A-A$ and $B-B$. Some relationship is obviously to be expected; it was not apparent earlier because incorrect radii had been assigned to three of the most electronegative elements. It is, however, relevant to inquire whether the same relationship should apply to all pairs of dissimilar atoms; that is, whether the nature of a bond is the same for any pair of atoms with a particular difference in electronegativity regardless of the absolute values of the electronegativity coefficients. It will also be necessary to inquire into the general validity of Pauling's curve relating the percentage ionic character of a bond to the difference between the electronegativities of the atoms, a curve based on the dipole moments of the halogen halides. For example, are we justified in assuming that two electrons are shared in exactly the same way between two fluorine atoms as between, say, two carbon atoms, and is the relation between bond type and electronegativity the same for atoms in a horizontal row as in a vertical column of the Periodic Table? The difference between the electronegativity coefficients of carbon and silicon is greater than that between those of, say, carbon and nitrogen, yet Pauling quotes the carbon-silicon distance in tetramethyl-silane as an example of the additivity of covalent radii.

In order that future discussions of the nature of bonds shall be put on a sound basis, it is to be hoped that some of these points and some of the experimental data will be critically examined. The results are of great general interest to many chemists, of whom relatively few may be in a position to criticize the conclusions reached by the specialist. This is one of the dangers attending the increasing specialization which is a characteristic of modern science. Perhaps there is still room for a Carneades, of whose function Boyle (in "The Sceptical Chymist") wrote, "that having thus drawn the chymists' doctrine out of their dark and smokie laboratories, either judicious men shall henceforth be allowed calmily and after due information to disbelieve it, or those abler chymists will be obliged to speak plainer than hitherto has been done, and maintain it by better experiments and arguments".

A. F. Wells.

\section{THE SCIENCE OF PLANT BREEDING}

\author{
By D. LEWIS \\ John Innes Horticultural Institution
}

$\mathrm{T}$ HAT breeding is an art and not a science is an opinion frequently expressed by many who are concerned with plant and animal improvement. Whatever they have meant by art in this generalization, it appears that, in their hands, breeding is mainly unscientific. It is certain, however, after reading Dr. Harland's report on cotton breeding in Peru ${ }^{1}$ that, in his hands, breeding is truly scientific and as such can give good results quickly. It is scientific because measurements of the relevant characters were his standards for selection, and because genetical principles were the guide in calculating the number of plants required, and in deciding the type of breeding. Therefore, he did not rely upon the hazards of hit-and-miss intuition of the art side of breeding.

Tanguis, originating as a chance seedling in 1908, has been the main variety of cotton grown in Peru since that date. It was originally of excellent quality and yield; but subsequent contamination from crossing and seed mixing with other stocks has caused much deterioration. In 1940 Harland had the task of reviving and improving its characteristic qualities, and in three years he has increased the mean fibre length by $\frac{8}{8}$ in. above the mean for commercial Tanguis, and has also made improvements in ginning percentage, boll weight, colour and yield. So that in $1943,2,100$ acres were planted with his improved seed.

How was this remarkable achievement brought about? The taxonomic position and origin of the variety Tanguis are first considered, since these are prerequisites to any scientific breeding programme. Tanguis has most of the characters of Gossypium barbadense with the early maturity of $G$. hirsutum, as a result of which previous Peruvian workers believed it to be a hybrid. But some early work of Harland showed that it is pure barbadense and that the early maturity arose by selection due to associa- 